\title{
SOME GROUPS THAT ARE JUST ABOUT FREE
}

\author{
BY GILBERT BAUMSLAG ${ }^{1}$
}

\author{
Communicated by Michio Suzuki, May 16, 1967
}

Introduction. I have recently come across a rather extraordinary class of groups $\mathcal{G}$ while looking for a group of cohomological dimension 1 which is not free. The groups $G \in \mathcal{G}$ are generated by three elements $a, b, c$ satisfying the single defining relation $a=c^{-i} a^{-1} c^{i} a \cdot c^{-j} b^{-1} c^{j} b$ :

$$
G=\left(a, b, c ; a=c^{-i} a^{-1} c^{i} a c^{-j} b^{-1} c^{j} b\right) \quad(i j \neq 0) .
$$

The purpose of this announcement is to make known the surprisingly similar behavior of these groups in $\mathcal{G}$ and the free group $F$ of rank two.

THEOREM. Every group $G$ in $\mathrm{g}$ satisfies the following conditions:

(i) $G($ like $F)$ is the third term of an exact sequence

$$
1 \rightarrow N \rightarrow G \rightarrow Z \rightarrow 1
$$

where $N$ is free and $Z$ is infinite cyclic;

(ii) the 2-generator subgroups of $G$ (like those of $F$ ) are free;

(iii) $G($ like $F)$ is residually nilpotent, i.e.

$$
\bigcap_{i=1}^{\infty} \gamma_{i} G=1 \text {, }
$$

where $\gamma_{i} G$ is the ith term of the lower central series of $G$;

(iv) $G / \gamma_{i} G \cong F / \gamma_{i} F$ for $i=1,2, \cdots$;

(v) $G / G^{\prime \prime} \cong F / F^{\prime \prime}$ where $X^{\prime \prime}$ is the second derived group of the group $X$;

(vi) $G$ is not free. ${ }^{2}$

Before making a few remarks about the proof of the Theorem I would like to point out that, by a theorem in [1], $G$ is of cohomological dimension at most 2 . Whether every group in $\mathcal{G}$ is of cohomological dimension precisely 2 , I do not as yet know!

Incidentally, groups satisfying (iii) and (iv) (termed parafree in [2]) are plentiful [2]. The main point of the theorem is that nonfree parafree groups $G$ satisfying (v) can exist.

Remarks on the proof of the theorem. Let $G$ be the group given by (1). We verify that $G$ has the properties (i)-(vi).

1 The author is a Sloan Fellow.

${ }^{2}$ I thank S. Meskin for helping to verify (vi). 
(i) is straightforward and is proved by taking $N$ to be the normal closure of $a$ and $b$ and applying the Reidemeister-Schreier procedure for finding generators and relations for a subgroup of a group given by generators and relations (see e.g. [3, p. 86]).

(ii) holds for all parafree groups [4].

(iii) is the most difficult property to verify. One proves that $G$ has this property by making use of the exact sequence (i).

(iv) and (v) follow exactly from the observation that $G$ is the freest group generated by $a, b$ and $c$ satisfying the relation $a^{-1} c^{-i} a^{-1} c^{i} a c^{-j} b^{-1} c^{j} b=1$.

(vi) There is an algorithm introduced by J. H. C. Whitehead [5] whereby one can effectively determine whether a group with a single defining relation is free. (vi) follows on applying this algorithm to $G$.

\section{REFERENCES}

1. R. C. Lyndon, Cohomology theory of groups with a single defining relation, Ann. of Math. (2) 52 (1950), 650-665.

2. G. Baumslag, Groups with the same lower central sequence as a relatively free group. I: The groups, Trans. Amer. Math. Soc. (to appear).

3. W. Magnus, A. Karrass and D. Solitar, Combinatorial group theory, Interscience, New York, 1966.

4. G. Baumslag, Groups with the same lower central sequence as a relatively free group. II: Properties, Trans. Amer. Math. Soc. (to appear).

5. J. H. C. Whitehead, On certain sets of elements in a free group, Proc. London Math. Soc. 41 (1936), 48-56.

Graduate Center, The City University of New York 